detailed questions about risk taking behaviours, specific types of physical training activities, and reasons CF members were unable to deploy for active duty. There were three separate outcomes of interest, acute injury, RSI and deployment prohibitive musculoskeletal injury. Multivariate logistic regression models were developed to investigate which injury risk factors remained significant when adjusted for other parameters in the model.

Findings Bivariate analysis identified several military and physical training variables significantly associated with acute, RSI and deployment prohibitive injuries. Multivariate logistic regression revealed more complex relationships; the association between training and injury was mediated by the inclusion of lifestyle and demographic variables in the models. This exploratory approach provides a more comprehensive description of injuries leading to more effective surveillance and prevention planning in the CF.

\title{
0558 KEY FACTORS ASSOCIATED WITH THE HIGH BURDEN OF INJURIES IN THE CANADIAN FORCES
}

E Payne* Correspondence: Department of National Defence, Government of Canada, 1745 Alta Vista Dr. Ottawa, Ontario K1A OK6, Canada

10.1136/ip.2010.029215.558

Background Military populations are particularly vulnerable to injury due the nature of their work and the required levels of physical training. The Canadian Forces Health and Lifestyle Information Survey (HLIS) is a regularly conducted population health survey on health status, risk factors and demographics from a stratified random sample of Canadian Forces (CF) personnel. The 2008/9 HLIS indicated that in the preceding year $23 \%$ of Canadian Forces (CF) personnel had sustained an activity limiting repetitive strain injury (RSI) and $21 \%$ an activity limiting acute injury. Among CF personnel unable to deploy, 32\% identified musculoskeletal injury as the reason.

Methods HLIS sampling methods are described in detail elsewhere. The 2008/2009 version of the HLIS included more 